\title{
A critical test of the failure-to-engage theory of task switching
}

\author{
SCOTT BROWN and CURTIS LEHMANN \\ University of California, Irvine, California \\ and \\ DANE POBOKA \\ University of Newcastle, Callaghan, Australia
}

\begin{abstract}
When people switch between two tasks, their performance on each is worse than when they perform that task in isolation. One theory of this "switch cost" is the failure-to-engage (FTE) theory, which posits that observed responses are a simple mixture of prepared and unprepared response strategies. The probability that participants use prepared processes can be manipulated experimentally (e.g., by changing preparation time). The FTE theory is a binary mixture model and therefore makes a strong prediction about the existence of fixed points in response time distributions. We found evidence contradicting this prediction, using data from 20 participants in a standard task-switching paradigm. In this article, we examine reasons for the failure of the FTE theory, and we demonstrate that a generalized version of FTE theory accommodates our data.
\end{abstract}

The study of executive control mechanisms has benefited in recent years from experiments in the task-switching paradigm (for a review, see Monsell, 2003). These experiments require participants to perform two different tasks (A and $\mathrm{B}$ ) repetitively, sometimes switching between the tasks and sometimes repeating a task. Rogers and Monsell (1995) developed a task-switching paradigm using alternating runs: Participants were required to perform tasks in a sequence, such as AABBAABB. This design ensured that both task switching (from A to B and from $B$ to $A$ ) and task repetition (AA and $\mathrm{BB}$ ) occurred within the same block of trials. Rogers and Monsell found a task-switching cost: Response time (RT) was slower on switch trials than on nonswitch trials. Rogers and Monsell also found that this switch cost decreased when the interval between one stimulus and the next (the response-to-stimulus interval, or RSI) was increased but that there was an irreducible switch cost that was not removed, no matter how long RSI became. Other researchers have since replicated and extended these findings, using the repeated runs design (e.g., De Jong, 2000; Gilbert \& Shallice, 2002; Karayanidis, Coltheart, Michie, \& Murphy, 2003; Lien, Schweickert, \& Proctor, 2003; Los, 1999; Nieuwenhuis \& Monsell, 2002; Sohn \& Anderson, 2003; Yeung \& Monsell, 2003).

\section{The Failure-to-Engage Hypothesis}

Many theories have been proposed to explain the costs associated with switching between tasks. Some theories

The authors thank Eric-Jan Wagenmakers for comments on an earlier draft. Correspondence should be addressed to S. Brown, Department of Cognitive Sciences, 3151 Social Sciences Plaza, University of California, Irvine, CA 92697-5100 (e-mail: scottb@uci.edu). use the concept of "task-set inertia" (TSI), which has garnered substantial empirical support (e.g., Allport, Styles, \& Hsieh, 1994; Gilbert \& Shallice, 2002; Los, 1999; Yeung \& Monsell, 2003). Theories including TSI propose that each of the two tasks to be performed has an associated task set, loosely defined as a mental state that must be prepared in order to accomplish that task. Switch costs are explained as at least partly due to inefficiency in changes between task sets. Other theories include the concept of stimulus-cued completion (SCC; Rogers \& Monsell, 1995). SCC posits that residual switch costs (i.e., switch costs that remain even at very long RSI values) are due to an inability to complete task set switching without the presence of an imperative stimulus.

The failure-to-engage (FTE) theory of De Jong (2000) took a different approach and assumed no difference between voluntary (endogenous) and stimulus-cued (exogenous) preparation. Rather, the FTE theory proposed that task preparation, however triggered, was all or none. Participants attempt to switch task sets before each switch trial, but sometimes this attempt fails. According to this account, responses arise from either a "prepared" process, in which the participant is ready to perform the task, or an "unprepared" process, in which the participant must first load the associated task set before the task can be performed (see Figure 1). Prepared and unprepared processes lead to faster and slower RTs, respectively. Thus, the observed RT on any given trial is a sample from either the distribution associated with the prepared process (with probability given by, say, $\alpha$ ) or the unprepared distribution (with probability $1-\alpha$ ). The observed RT distribution is a mixture of two unobserved distributions.

The FTE theory does not specify the form of the unobserved distributions or the processes that give rise to them, 


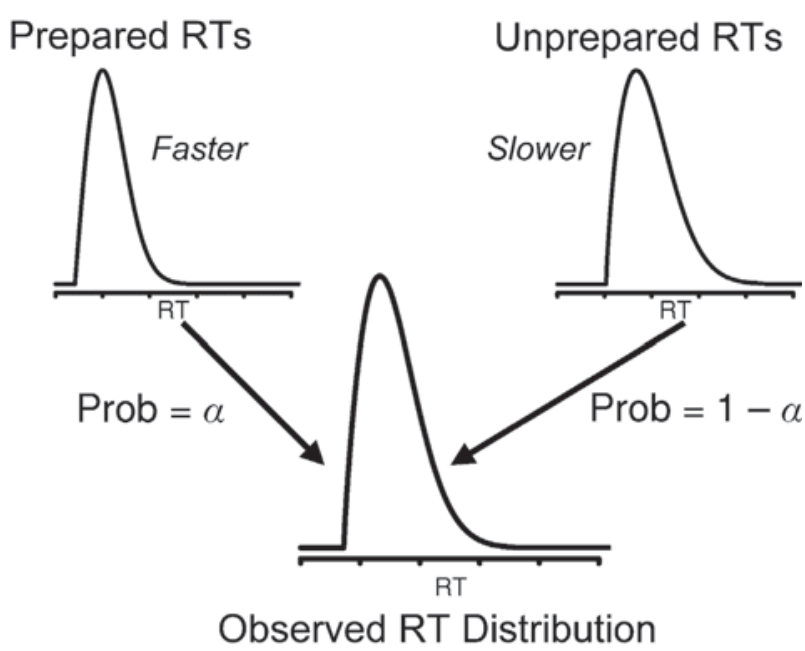

Figure 1. Schematic illustration of the failure-to-engage theory of task switching. Observed response time (RT) distribution is a mixture of two unobserved distributions: those from "prepared" and those from "unprepared" mental states.

but it does provide constraints on the value of $\alpha$, the mixing parameter. In situations in which the participant is relatively unprepared, $\alpha$ will be small, and many observed responses will be drawn from the unprepared distribution. When the probability of preparation (i.e., $\alpha$ ) increases, the observed distribution is more similar to the prepared RT distribution.

According to the FTE theory, the probability of preparation $(\alpha)$ can be changed by experimental manipulations. For example, longer RSI values or greater rewards for fast responses should both increase $\alpha$. Nieuwenhuis and Monsell (2002) found that larger RSI values increased the value of $\alpha$ in model fits of the FTE theory, but there was a limit: Across participants, the mean estimate of $\alpha$ was only $64 \%$. This implies that residual switch costs are due to participants' failure to engage on over one third of trials, even when highly motivated and given ample preparation time.

De Jong (2000) and Nieuwenhuis and Monsell (2002) found that the FTE model fit the observed data well, lending support to that theory. Other researchers have also observed data that are generally consistent with the FTE theory or, at least, are not in direct contradiction to it. Lien et al. (2003) found evidence supporting the assumption that executive control processes can accommodate processing of only one task at a time, consistent with the mixture assumption of FTE. Los (1999) found evidence in favor of models that include two separate response strategies, such as the FTE theory (as opposed to criterion shifting models). Monsell, Sumner, and Waters (2003) observed that RT distributions were generally consistent with predictions from the FTE model, although that model was not uniquely supported by their data.

\section{A Critical Test of the FTE Theory}

Other results have been less clear in their support of FTE theory. Gilbert and Shallice (2002) found that the binary assumption of FTE (i.e., that task-set preparation is "all or nothing") was not required to explain their data. Lien et al. (2003) observed interactions between RSI and task response style that were inconsistent with the basic version of FTE theory. These studies did not directly test the FTE model: They were designed to meet some other goals, and support (or not) for the FTE theory was observed only indirectly. Nieuwenhuis and Monsell's (2002) study was designed as a direct test of the FTE theory, but the test was an evaluation of goodness of fit: Data were collected, and the FTE theory was shown to be able to fit them. However, goodness-of-fit tests are subject to a common criticism: Just because a model is consistent with some data does not mean that the model is "correct," especially when it is not clear what other patterns of data are consistent with the model (e.g., Roberts \& Pashler, 2000).

A better way to test the FTE hypothesis is to derive a strong prediction that can be tested in data. Falmagne (1968) demonstrated a surprising prediction of all binary mixture models - their distributions have fixed crossing points across changes in mixture probabilities. Falmagne (1968) used this property to disprove another binary mixture model of choice RT (Falmagne's, 1965, "fast guess" theory). The fixed-point property applies to any binary mixture model, including the FTE theory. Falmagne's observation was that, if the probability distribution functions (PDFs) of the two mixture components cross at some point, then all mixtures of those two distributions must also cross at that same point. ${ }^{1}$ For the FTE theory, this means that if the PDFs for prepared and unprepared RTs cross at some point, then all observed RT distributions - mixtures from those two - must also cross at the same point. This property is illustrated in cartoon fashion in Figure 2.

If the FTE theory is correct, when an experimental manipulation varies, the probability of preparation $(\alpha)$ the PDFs produced under different levels of that manipulation must share a common crossing point, such as the $25 \%, 50 \%$, and $75 \%$ mixtures in Figure 2. This property leads to a simple critical test for the FTE theory: Experimentally manipulate the probability of preparation and observe the crossing points of the resulting PDFs. If a binary mixture process underlies RT, the PDFs must share common crossing points.

This test applies only to theories that are pure binary mixtures. That is, the fixed-point prediction will hold only for theories that assume the same two unobserved distributions mix in different proportions. There must be no other change than mixing probability between the different conditions; otherwise, the fixed-point property will not hold. Although these restrictions may seem unnecessarily strict, the FTE theory has been developed and implemented as a pure binary mixture model, with no model changes other than mixture probability between different conditions (e.g., De Jong, 2000; Nieuwenhuis \& Monsell, 2002). Relaxation of these strict assumptions can allow the FTE theory to avoid making the fixed-point prediction (we examine such a generalization of the FTE theory later). Note also that De Jong's inclusion of the $\delta$ parameter (p. 361) does not change the fixed-point prediction. This parameter captures the idea that the (hypothetical) 


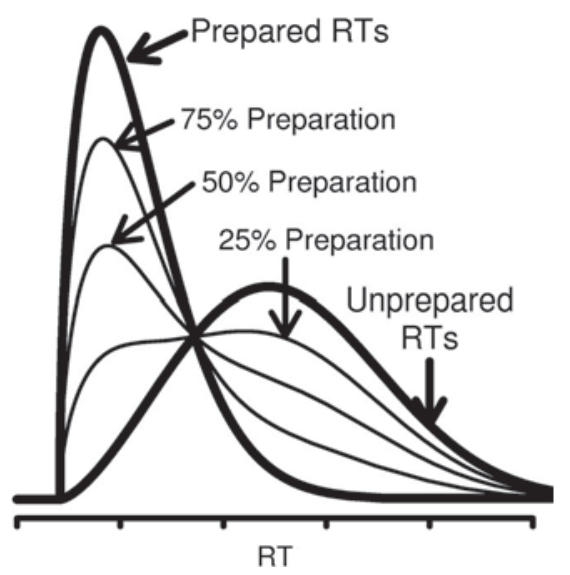

Figure 2. Falmagne's (1968) fixed-point property as it applies to the FTE theory. PDFs produced from different levels of preparedness must share a common crossing point.

mixing distribution for "prepared" responses is related by a simple shift to the (observed) distribution for nonswitch responses in the easiest condition. This assumption simply specifies the form of one of the mixing distributions, but the fixed-point property holds for any particular forms of the mixing distribution.

We carried out the test below using a standard taskswitching paradigm with a letter task and a digit task. For each task, we collected data from switch and nonswitch trials, each for three different RSI values. The FTE theory supposes that all six of these distributions arise as different $\alpha$ mixtures of the same pair of underlying distributions (for each task). The six distributions had 15 possible pairings, corresponding to 15 crossing points, and 12 of these were predicted to be identical under the FTE theory. If the FTE theory is correct, those 12 crossing points should not vary systematically.

\section{METHOD}

\section{Participants}

Twenty undergraduate students (ages 18-46 years, $M=23.4$ years) from the University of Newcastle participated in this study in exchange for partial course credit.

\section{Task and Stimuli}

The design was based on the alternating-runs paradigm used by Rogers and Monsell (1995). Two tasks were used: a letter classification task (Task L) and a digit classification task (Task D). The participants classified letters as either a vowel (A, E, I, U) or a consonant $(\mathrm{G}, \mathrm{K}, \mathrm{M}, \mathrm{R})$ and digits as either odd $(3,5,7,9)$ or even $(2$, $4,6,8)$. The stimuli for every trial consisted of a character randomly sampled from both task sets (e.g., A3). Within a block of trials, responses to the two tasks could be either congruent (both characters required the same buttonpress) or incongruent (each character required a different buttonpress). Congruent responses occurred only $12.5 \%$ of the time. Each response was made with an index finger, counterbalanced across participants. Responses were recorded using the left and right mouse buttons.

Trials moved clockwise around a $2 \times 2$ grid in a DDLLDDLL $\ldots$ sequence. The appropriate task was indicated by the location of the current trial within the grid (e.g., letter task = top squares; digit task $=$ bottom squares), with the location to task mapping counterbalanced across participants. Stimulus characters were presented in a white font on a black background on a 15 -in. monitor at a viewing distance of $60 \mathrm{~cm}$. The $2 \times 2$ grid was 30 characters wide and 13 characters high. Square quadrants were defined within the grid.

Three RSIs were used for this experiment: 150,600 and $1,200 \mathrm{msec}$. The experiment was divided into two sessions: a blocked RSI session and a random RSI session. In the blocked session, the RSI remained constant within each block of trials (cf. Rogers \& Monsell, 1995, Experiment 3), and each RSI block occurred twice for six blocks in total. The order of RSI presentation was counterbalanced across participants. In the random session, RSI varied within a block of trials (cf. Rogers \& Monsell, 1995, Experiment 2), and all three RSI values were presented equally often in a random order. Six blocks were presented during this session. In total, the experiment comprised 12 blocks with 100 trials each (4 warm-up +96 experimental trials). Each block contained an equal number of trials for type (switch or nonswitch) and task (letter or digit).

\section{Procedure}

Each participant completed a $30-$ min practice session prior to the 50-min experimental session. The practice session included two shorter blocks ( 50 trials) of both tasks separately (letter task then digit task). Task instructions were displayed before each block and were visible during the practice trials. After each block, performance feedback was displayed, including accuracy and mean RT. Next, two blocks (600-msec RSI followed by 150 -msec RSI) of task-switch trials were presented (100 trials). During these trials, only the taskrelevant character was displayed on the screen (i.e., only one character was used for each trial). The order of the remaining practice trials depended on session type (blocked or random). For the blocked session, two blocks (1,200-msec RSI followed by 150 -msec RSI) of standard alternating-runs switch trials were presented (50 trials each). The stimuli for these trials were composed of characters from both task sets. For the random session, one block with randomly ordered RSIs of standard alternating-runs switch trials was presented (100 trials). All three RSIs were used for this block. Only the final practice block type was run at the start of the second session.

Prior to each block of the experimental session, instructions were displayed reminding the participants of the correct response mappings. The instructions were not visible during the experimental trials. Stimuli for the first 4 (warm-up) trials for each block were randomly chosen. Each stimulus was presented on the screen until a response was recorded or until 5,000 msec had passed. If an incorrect response was recorded or 5,000 msec had elapsed, a brief tone $(750 \mathrm{~Hz})$ sounded for $200 \mathrm{msec}$ before the onset of the following task. The participants were encouraged to prepare for the upcoming task and to respond to the task-relevant character as soon as possible while maintaining high accuracy. Feedback on performance for the previous three blocks was given at the end of each block. The participants were encouraged to take short breaks at the end of each block if they wished.

\section{RESULTS}

To test the fixed-point property, we needed to estimate RT distributions. This required stationary data, to meet the assumption of standard estimation procedures. We carefully censored our data to help meet this assumption. Data from the practice session were discarded, as were data from the first 4 trials in each block of the experimental session. We also censored RTs associated with incorrect responses and RTs from responses immediately following an error. Finally, our experiment included several blocks in which different RSI values were randomly mixed across trials. These were included in the experiment for a differ- 
ent purpose; hence, they are not analyzed below. Mixed RSI blocks typically lead to different patterns of switch cost reduction than do uniform RSI blocks (e.g., see Experiments 2 and 3 of Rogers \& Monsell, 1995). After censoring, each participant contributed between 833 and 1,134 data points, or around 70 to 95 data points per RT distribution, to the analyses below.

\section{Task-Switching Effects}

As expected, longer RSI was associated with smaller RT and with lower switch costs (see Table 1).

For the letter task, the mean switch cost in RT decreased from $379 \mathrm{msec}$ at the shortest RSI to $334 \mathrm{msec}$ at the medium RSI and $265 \mathrm{msec}$ at the longest. For the digit task, the switch costs decreased from $441 \mathrm{msec}$ to $354 \mathrm{msec}$ and then to $251 \mathrm{msec}$. These effects were statistically reliable: A repeated measures ANOVA $^{2}$ showed a main effect of RSI $\left[F(2,38)=6.7, M S_{\mathrm{e}}=10,149, p<.01\right]$, a main effect of switch versus nonswitch trials $[F(1,19)=$ $\left.89, M S_{\mathrm{e}}=37,841, p<.001\right]$, and an interaction effect $\left[F(2,38)=16, M S_{\mathrm{e}}=3,545, p<.001\right]$. There was also a tendency for nonmonotonicity with RSI in RT for nonswitch trials. This tendency was not significant but did appear in both letter and digit tasks (and has been seen in other studies; e.g., Monsell, 2003, Figure 2).

The accuracy data in Table 2 show that the participants were able to maintain a relatively constant accuracy rate across the two tasks and across RSI, as instructed. There was a large and reliable switch cost in accuracy [94.3\% for switch trials vs. $97.9 \%$ for nonswitch trials; $F(1,19)=$ $\left.31, M S_{\mathrm{e}}=0.001192, p<.001\right]$. No other ANOVA effects were significant for accuracy data. This shows that the participants were relatively successful at maintaining a constant error rate across tasks and RSI, forcing effects into RT rather than accuracy.

Figure 3 shows cumulative distribution functions (CDFs) averaged across participants. These functions show similar patterns to those previously observed by De Jong (2000, his Figure 15.2) and others-patterns that have been interpreted as support for the FTE theory. In both letter and digit tasks, the CDFs corresponding to the long- and medium-RSI switch conditions (solid lines, filled triangles, and circles) begin closest to the nonswitch CDFs in the lower left corner of the plot, and they end closest to the long-RSI switch conditions in the upper right corner. This pattern is consistent with a mixture model: The fastest responses in these two intermediate conditions are similar to the fastest responses in the fast- est condition, and the longest responses are similar to the longest responses in the slowest condition.

Although the patterns observed in Figure 3, and by others, are consistent with a mixture model such as the FTE theory, they do not provide unequivocal support. These CDFs are averaged across participants. This averaging process could have distorted the shape of the CDFs, unless the individual CDFs all belonged to the same locationscale family (see Brown \& Heathcote, 2003).

\section{Distribution Crossing Points}

All distribution-estimation and crossing-point analyses were carried out on unaveraged, individual data. For each participant, data were separated into 12 conditions using factorial combinations of digit tasks and letter tasks, switch trials and nonswitch trials, and the three RSI levels. For each of these 12 data conditions, separately for each participant, an empirical estimate of the PDF was calculated using a standard kernel density estimator. Kernel density estimation can be thought of as a continuous version of the histogram. The most succinct description is mathematical: Given data $\left\{x_{1}, \ldots, x_{N}\right\}$, the height of the density estimate at point $t$ is given by

$$
\frac{1}{N} \sum_{i=1}^{N} f\left(\frac{t-x_{i}}{h}\right) .
$$

The value $h$ refers to a bandwidth, and it governs the amount of smoothing - analogous to the bin width of a histogram. The function $f$ refers to the kernel and can be any density function with unit integral (we chose the standard normal density). A comparison with standard histogram estimation may make this equation clearer for the reader new to density estimation. When constructing a histogram, each observation, $x_{i}$, adds one unit of height to the histogram in the binning interval covering that observation. A kernel density estimator makes the notion of bin intervals continuous and graded, rather than discrete and absolute. So observation $x_{i}$ will add quite a lot of height to the estimated density at the value $x_{i}$ and add less height at values nearby and will have almost no effect at values far from $x_{i}$. Standard texts on kernel density estimation are plentiful (see, e.g., Fan \& Gijbels, 1996; Silverman, 1986; or Van Zandt, 2000, for an introduction specific to RT distributions).

For each condition, we calculated density estimates at 1,024 evenly spaced points between 400 and $2,000 \mathrm{msec}$ using a Gaussian kernel with a bandwidth 1.25 times the

Table 1

Mean Response Times (in Milliseconds) and Standard Deviations (SDs)

\begin{tabular}{|c|c|c|c|c|c|c|c|c|}
\hline \multirow[b]{3}{*}{ RSI (msec) } & \multicolumn{4}{|c|}{ Letter Task } & \multicolumn{4}{|c|}{ Digit Task } \\
\hline & \multicolumn{2}{|c|}{ Switch Trial } & \multicolumn{2}{|c|}{ Nonswitch Trial } & \multicolumn{2}{|c|}{ Switch Trial } & \multicolumn{2}{|c|}{ Nonswitch Trial } \\
\hline & $M$ & $S D$ & $M$ & $S D$ & $M$ & $S D$ & $M$ & $S D$ \\
\hline 150 & 1,135 & 560 & 756 & 394 & 1,197 & 612 & 756 & 362 \\
\hline 600 & 1,056 & 546 & 722 & 329 & 1,052 & 559 & 698 & 322 \\
\hline 1,200 & 1,027 & 511 & 762 & 362 & 1,028 & 541 & 777 & 412 \\
\hline
\end{tabular}

Note-RSI, response-to-stimulus interval. 
Table 2

\begin{tabular}{cccccc}
\multicolumn{5}{c}{ Mean Accuracy (\%) } \\
\hline & \multicolumn{2}{c}{ Letter Task } & & \multicolumn{2}{c}{ Digit Task } \\
\cline { 2 - 3 } \cline { 5 - 6 } RSI $(\mathrm{msec})$ & Switch Trial & Nonswitch Trial & & Switch Trial & Nonswitch Trial \\
\hline 150 & 95.3 & 99.0 & & 93.6 & 97.5 \\
600 & 96.2 & 92.8 & & 98.0 & 96.8 \\
1,200 & 94.6 & 93.7 & & 98.8 & 97.0 \\
\hline
\end{tabular}

Note-RSI, response-to-stimulus interval.

value given by Silverman's (1986, p. 48) "rule of thumb" automatic bandwidth selector. This automatic bandwidth selection avoids subjective bias inherent in manual selection. The factor of 1.25 provided some "over smoothing," which helped minimize problems associated with multiple PDF crossing points. Figure 4 shows estimated density functions averaged across all participants. The crossing points in these averaged plots should not be interpreted too carefully: Averaging such nonlinear functions across participants can cause great distortions in functional form and, hence, crossing-point locations. There is no method available for averaging density functions that avoids these problems of distortion (see, e.g., Brown \& Heathcote, 2003; Rouder \& Speckman, 2004). It is tempting to draw conclusions from the averaged PDF plots: For instance, in the consonant-vowel task (left plot), it seems that the short-RSI nonswitch trials (solid gray line) are shifted longer relative to the medium-RSI nonswitch trials (dashed line) by about $50 \mathrm{msec}$. This effect would also shift the crossing points for the short-RSI nonswitch condition with the other switch distributions - an effect that is incompatible with a binary mixture model such as the FTE. However, it is dangerous to interpret such highly averaged data; therefore, we focus below on analyses of crossing points calculated from individual participants' PDFs only.

After estimating the 12 densities for each participant, we located the distribution crossing points. For each task (letter and digit), there were six distributions, leading to 15 unique distribution pairs for which crossing points could be calculated. We used only 12 of these: The FTE theory may predict perfect engagement on nonswitch trials, so the three different RSI conditions may be predicted to lead to identical nonswitch RT distributions and, hence, ill-defined crossing points.

It was possible that each of the remaining 12 distribution pairs for the letter task and the digit task had multiple crossing points. To overcome this, we always selected the crossing point closest to the midpoint between the modes of the estimated distributions. The midpoint between modes is associated with higher data density and, hence, better estimation accuracy. Choosing the cross point this way also avoids a problem that arises when distributions are very similar. If two distributions are identical, their crossing point is any arbitrary value at all. If the distributions are very similar, they will cross at numerous points. Our use of a fixed algorithm to choose just one of those points in a reliable manner reduces this variability. This avoids the identification of spurious differences in cross points due to comparison of very similar distributions. Figure 5 shows the averages of the 12 crossing points for the letter and digit tasks, with error bars corresponding to standard error of the mean (SEM) across participants.

There appears to be large and systematic variability in the crossing points illustrated in Figure 5. For example, the crossing points of shorter RSI switch distributions with longer RSI nonswitch distributions (horizontally shaded bars) are always longer than other crossing points. We calculated a two-way repeated measures ANOVA on the estimated crossing points, using digit/letter and distribution pair (the 12 conditions in Figure 5) as factors. Cross points for letter data were a little faster $(837 \mathrm{msec})$
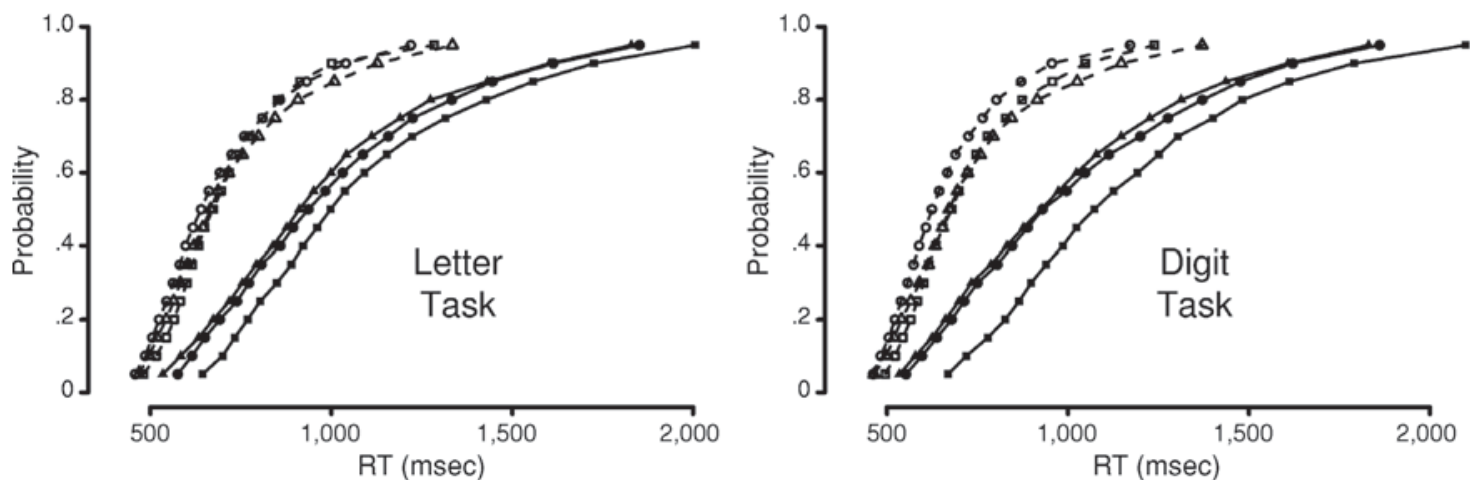

Figure 3. Averaged cumulative distribution functions. Quantile RTs $(5 \%, 10 \%, \ldots, 95 \%)$ were calculated for each subject separately and then were averaged. Solid lines and filled symbols represent data for switch trials; dashed lines and open symbols represent data for nonswitch trials. Data for the short-RSI condition are plotted with squares; data for the medium-RSI condition are plotted with circles; data for the long-RSI condition are plotted with triangles. 

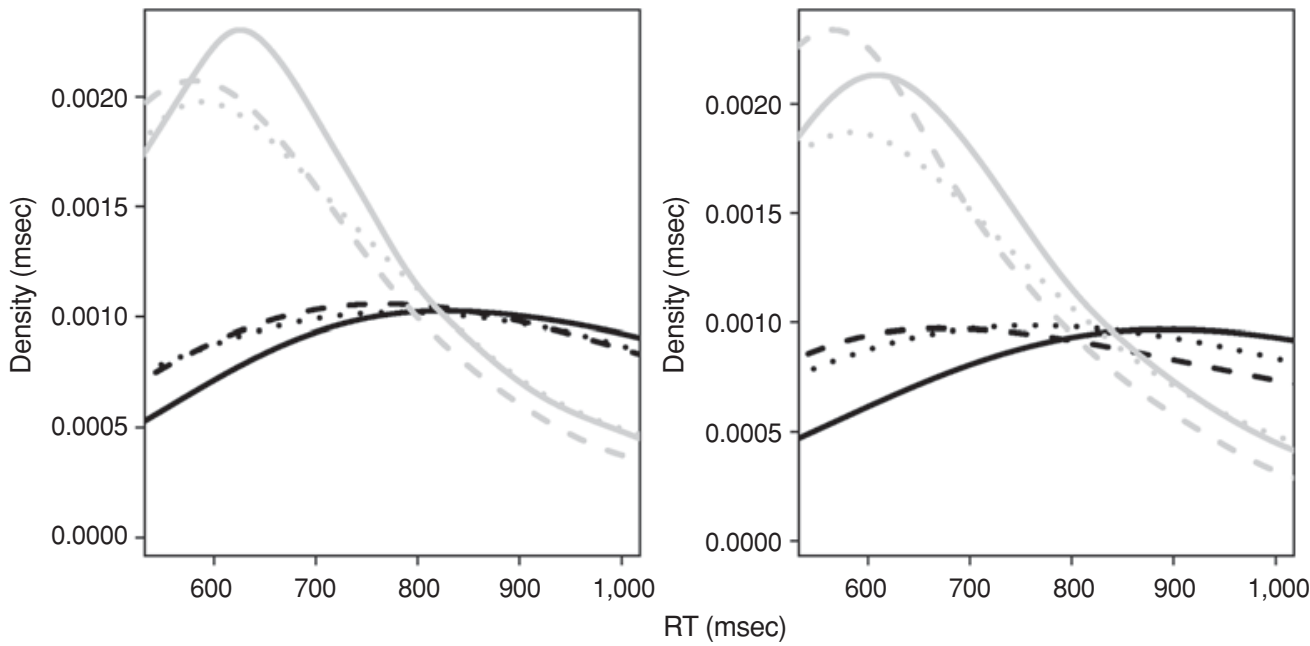

Figure 4. Density estimates averaged across participants. The left panel shows letter task data; the right panel shows digit task data. Distributions from nonswitch trials are shown in gray; distributions from switch trials are shown in black. Long-RSI $(1,200-\mathrm{msec})$ distributions are drawn with dotted lines; medium-RSI (600-msec) distributions are drawn with dashed lines; short-RSI (150-msec) distributions are drawn with solid lines.

than for digit data $(858 \mathrm{msec})$, but this trend was not significant $\left[F(1,19)<1, M S_{\mathrm{e}}=100,611\right]$. Confirming the trend from Figure 5, the effect of distribution pair was highly significant $\left[F(11,209)=3.6, M S_{\mathrm{e}}=27,841, p<\right.$ $.01]$. There was no significant interaction $[F(11,209)<1$, $\left.M S_{\mathrm{e}}=17,856\right]$. Separate one-way ANOVAs for the digit task and the letter task showed that the crossing-point differences were significant for both [digit task, $F(11,209)=$ $2.8, M S_{\mathrm{e}}=27,756, p<.01$; letter task, $F(11,209)=2.2$, $\left.M S_{\mathrm{e}}=20,941, p<.05\right]$.

Finally, we calculated a simpler, but less powerful, test of whether the cross points differed for the different distribution pairs. ${ }^{3}$ As a referent, we calculated the cross point of the fastest distribution (nonswitch, RSI $=1,200 \mathrm{msec}$ ) with the slowest distribution ( switch, RSI $=150 \mathrm{msec}$ ). This pair of distributions was most widely separated, and so these cross points were subject to smaller estimation error. Then, for each of the remaining 11 distribution pairings, one can ask whether those cross points fall systematically above or below the referent cross points. If the FTE theory is correct, and the distributions arise from a binary mixture, then the crossing points should be identical and the remaining cross points should fall equally often above and below the referent points, due to measurement noise. According to the binomial distribution, there is less than $p=.05$ combined probability of observing fewer than 8 or more than 13 cross points falling above the referent. In our data, we observed cross points falling above the
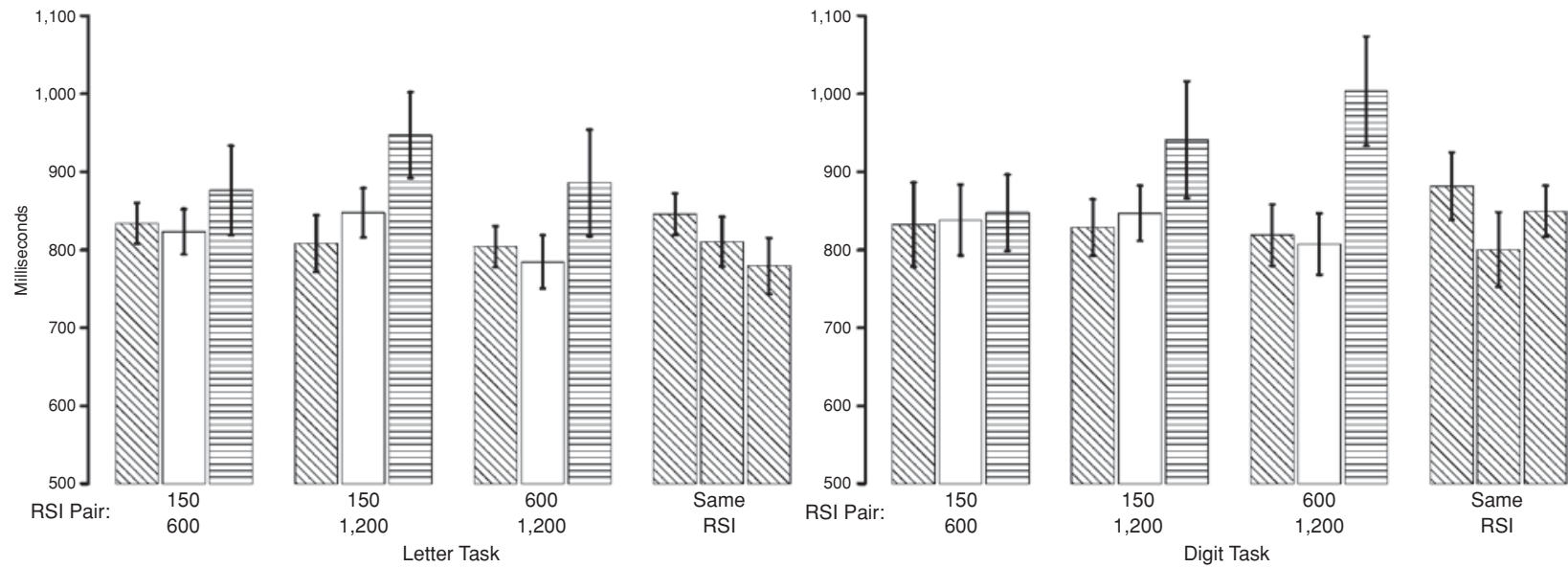

Figure 5. Mean crossing-point RT (in milliseconds) for 12 distribution pairs each from the letter task and the digit task. Error bars show $\pm S E M$ across participants. Abscissa labels give RSI values for each member of each distribution pair. Diagonal shading lines correspond to crossing points of switch with switch distributions. Unshaded bars correspond to longer RSI nonswitch distribution with shorter RSI switch distributions; horizontal lines correspond to longer RSI switch distributions with shorter RSI nonswitch distributions. 
referent for 3 of the 24 distribution pairs ( 2 of 11 for letter data and 1 of 11 for digit data) and below the referent for 1 distribution pair (in the digit task). Observing these 4 of 24 instances outside the $95 \%$ confidence interval is more than the rate expected by chance.

\section{DISCUSSION}

The analysis of distribution crossing points provides evidence against the FTE model of task switching. If taskswitching behavior actually was generated by a simple binary mixture process, the observed distributions would all have crossed at a common RT value, and the ANOVAs would have shown no significant effects of distribution pair. This was not the case. There were highly significant overall effects of distribution pair on crossing point, and these effects were reliable even when analyzing crossing points from the digit task or letter task alone. The FTE model cannot accommodate the present results without modification. There are several ways the model could be changed to avoid making the fixed-point prediction. For example, Falmagne's (1968) fixed-point property applies only to binary mixture models. If the mixture mechanism in the FTE model included more than just the two states of "prepared" and "unprepared," our test would not apply.

\section{Rescuing the Mixture Assumption}

The most interesting aspect of the FTE theory is the assumption that preparation for task switching is all-or-none and, hence, that observed RT distributions are formed by a binary mixture process. Falmagne's (1968) fixed-point test rules out the simplest binary mixture model but does not rule out the all-or-none assumption, because other, more complex, binary mixture models may still be tenable. The fixed-point test applies only to the simplest form of the FTE theory, which is the version currently in use (De Jong, 2000; Nieuwenhuis \& Monsell, 2002). This version of the FTE theory asserts that nothing changes between different RSI conditions or between switch and nonswitch trials - conditions except for the mixture probability, $\alpha$. Relaxing this assumption frees the FTE theory from making the strict fixed-point prediction. Below, we examine an extension of the FTE theory that makes a less stringent fixed-point prediction-a prediction supported by our data.

It is common in RT paradigms to observe shifts in RT distributions with variable foreperiods ${ }^{4}$ (see, e.g., Luce, 1986). Thus, it may be reasonable to generalize the FTE theory to allow the prepared and unprepared mixing distributions to shift with RSI (we will call the FTE theory when amended in this manner the shift-FTE theory). The shift-FTE theory does not make the fixed-point prediction of Falmagne (1965). For each task (letter and digit), the shift-FTE theory can be defined by two extra parameter values: a shift for the medium-RSI condition relative to the short-RSI condition, and a shift for the long-RSI condition relative to the short-RSI condition. If the shift sizes are known, the observed data can be "unshifted" by subtraction. These unshifted data then are subject to the fixed-point test of Falmagne, even though the raw data are not. This provides a stringent test of the shift-FTE theory.

We tested the shift-FTE theory in our data by estimating the shift parameters and then testing for the fixed-point property in the unshifted data. We estimated four shift parameters separately for each participant. These parameters describe the shift of the mixing distributions for the medium- and long-RSI conditions relative to the shortRSI condition, separately for the letter and digit tasks. We estimated the shift parameters using a simplex search (Nelder \& Mead, 1965) to minimize the variability of the crossing points in the unshifted data. In the letter task, the average (across participants) estimated shift parameter for the medium-RSI condition was $14 \mathrm{msec}$ (i.e., the prepared and unprepared mixing distributions were assumed to be $14 \mathrm{msec}$ faster in the medium-RSI condition relative to the short-RSI condition). The long-RSI mixing distributions were estimated to be an average of $8.6 \mathrm{msec}$ slower than the short-RSI mixing distributions. Note that this estimated slowdown in the long-RSI condition matches the observed slower mean RT for long-RSI nonswitch trials (see Table 1). For the letter task, the mixing distributions for the medium-RSI condition were estimated to be almost identical to those for the short-RSI condition, with an average shift of only $1 \mathrm{msec}$. The long-RSI mixing distributions were estimated to be $25 \mathrm{msec}$ slower than the short-RSI mixing distributions.

Although the mean estimated shift parameters were small, they were sufficient to rescue the shift-FTE theory and allow it to accommodate the observed pattern of crossing points. We calculated the crossing points for the unshifted data in the same manner as above, after subtraction of the estimated shift parameters from the observed data. Figure 6 shows the mean crossing points for the unshifted data, using the same format and $y$-scale as in Figure 5. The crossing points for the unshifted data appear to at least approximately satisfy Falmagne's (1965) fixed-point property - the variability in bar heights from Figure 6 is much smaller than the raw data crossing points from Figure 5.

As with the raw data above, we calculated a two-way ANOVA (distribution pair/letter vs. digit task) on the crossing points from the unshifted data. There was no significant variability in the crossing points attributed to any of the ANOVA terms [main effect of letter vs. digit task, $F(1,19)=1.1, M S_{\mathrm{e}}=103,170, p>.1$; main effect of distribution pair, $F(11,209)<1, M S_{\mathrm{e}}=29,985$; interaction, $\left.F(11,209)<1, M S_{\mathrm{e}}=18,119\right]$. The separate one-way ANOVAs for the digit task and the letter task both showed nonsignificant effects of distribution pair this time (both $F_{\mathrm{s}}<1$ ). We also recalculated the binomial test used above and found a marked improvement. We observed only 2 of 24 distribution pairs outside the $95 \%$ confidence interval. This is in contrast to the 4 of 24 observed for the raw data and is close to the rate expected by chance (1.2 of 24).

Our analysis of the shift-FTE theory demonstrates that the all-or-none preparation hypothesis of FTE is still tenable, as is the accompanying mixture hypothesis. There are many other ways the FTE theory could be amended to 

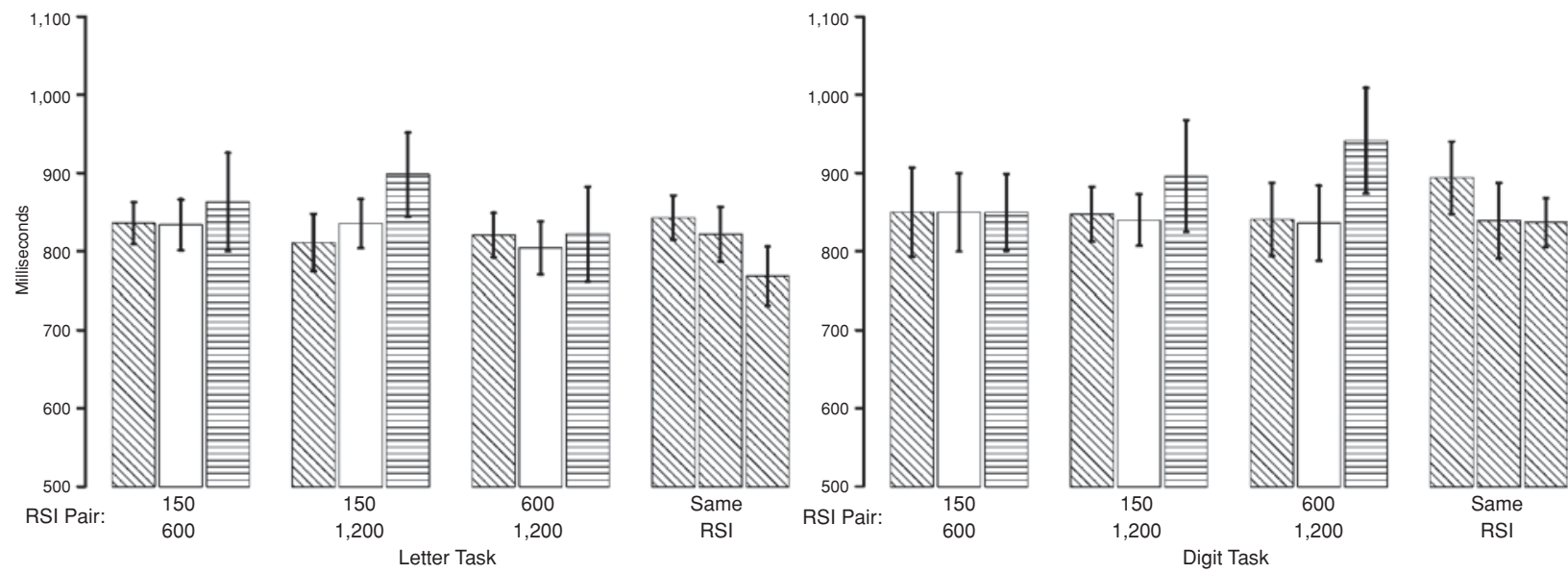

Figure 6. Mean crossing-point RT (in milliseconds) for 12 unshifted distribution pairs each from the letter task and the digit task. Error bars show $\pm S E M$ across participants. Order of the bars is the same as that in Figure 5.

accommodate our findings - perhaps by the addition of a third process or by allowing distribution shape, rather than location, to vary with RSI. Each of these changes to the FTE theory can be tested, as above, by estimating the distribution change parameters and then applying Falmagne's (1968) fixed-point test. The shift-FTE theory can be tested even more directly, however. This theory predicts that the basic fixed-point property of Falmagne will hold true if the mixing probability $(\alpha)$ is manipulated by some experimental manipulation other than RSI. For example, a future experiment could adjust mixing probability by changes in rewards or other incentives, and the shift-FTE theory would predict a fixed point in the observed distributions.

\section{REFERENCES}

Allport, D. A., Styles, E. A., \& Hsieh, S. (1994). Shifting intentional set: Exploring the dynamic control of tasks. In C. Umiltà \& M. Moscovitch (Eds.), Attention and performance XV: Conscious and nonconscious information processing (pp. 421-452). Cambridge, MA: MIT Press.

Brown, S., \& Heathcote, A. (2003). Averaging learning curves across and within participants. Behavior Research Methods, Instruments, \& Computers, 35, 11-21.

DE Jong, R. (2000). An intention-activation account of residual switch costs. In S. Monsell \& J. Driver (Eds.), Control of cognitive processes: Attention and performance XVIII (pp. 357-376). Cambridge, MA: MIT Press.

Falmagne, J. C. (1965). Stochastic models for choice reaction time with applications to experimental results. Journal of Mathematical Psychology, 2, 77-127.

FALMAGNe, J. C. (1968). Note on a simple fixed-point property of binary mixtures. British Journal of Mathematical \& Statistical Psychology, 21, 131-132.

FAN, J., \& GiJBeLs, I. (1996). Local polynomial modelling and its applications. London: Chapman \& Hall.

Gilbert, S. J., \& Shallice, T. (2002). Task-switching: A PDP model. Cognitive Psychology, 44, 297-337.

Karayanidis, F., Coltheart, M., Michie, P. T., \& Murphy, K. (2003). Electrophysiological correlates of anticipatory and poststimulus components of task switching. Psychophysiology, 40, 329-348.

Lien, M.-C., Schweickert, R., \& Proctor, R. W. (2003). Task-switching and response correspondence in the psychological refractory period paradigm. Journal of Experimental Psychology: Human Perception \& Performance, 29, 692-712.
Los, S. A. (1999). Identifying stimuli of different perceptual categories in mixed blocks of trials: Evidence for cost in switching between computational processes. Journal of Experimental Psychology: Human Perception \& Performance, 25, 3-23.

LuCE, R. D. (1986). Response times: Their role in inferring elementary mental organization. Oxford: Oxford University Press.

Monsell, S. (2003). Task switching. Trends in Cognitive Sciences, 7, 134-140.

Monsell, S., Sumner, P., \& Waters, H. (2003). Task-set reconfiguration with predictable and unpredictable task switches. Memory \& Cognition, 31, 327-342.

Nelder, J. A., \& Mead, R. (1965). A simplex algorithm for function minimization. Computer Journal, 7, 308-313.

Nieuwenhuis, S., \& Monsell, S. (2002). Residual costs in task switching: Testing the failure-to-engage hypothesis. Psychonomic Bulletin \& Review, 9, 86-92.

Roberts, S., \& Pashler, H. (2000). How persuasive is a good fit? A comment on theory testing. Psychological Review, 107, 358-367.

Rogers, R. D., \& Monsell, S. (1995). Costs of a predictable switch between simple cognitive tasks. Journal of Experimental Psychology: General, 124, 207-231.

Rouder, J. N., \& SPeckman, P. L. (2004). An evaluation of the Vincentizing method of forming group-level response time distributions. Psychonomic Bulletin \& Review, 11, 419-427.

Silverman, B. W. (1986). Density estimation. London: Chapman \& Hall. SoHn, M.-H., \& ANDERSON, J. R. (2003). Stimulus-related priming during task switching. Memory \& Cognition, 31, 775-780.

VAN ZANDT, T. (2000). How to fit a response time distribution. Psychonomic Bulletin \& Review, 7, 424-465.

Yeung, N., \& MonselL, S. (2003). The effects of recent practice on task switching. Journal of Experimental Psychology: Human Perception \& Performance, 29, 919-936.

\section{NOTES}

1. The proof is elementary: Suppose the PDFs of two mixing distributions are $f(x)$ and $g(x)$, and $f(y)=g(y)$ for some $y$. The PDF of an $\alpha$ mixture of $f$ and $g$ is given by $w(x)=\alpha f(x)+(1-\alpha) g(x)$, and so $w(y)=$ $\alpha f(y)+(1-\alpha) g(y)=f(y)$, by assumption.

2 . We collapsed across letter and digit task data for simplicity of exposition. Similar results were obtained on data from each task separately.

3 . We thank an anonymous reviewer for suggesting this.

4. We thank W. Trammell Neill for reminding us of this fact.

(Manuscript received May 19, 2004; revision accepted for publication May 29, 2005.) 